\title{
Spatial Correlation and Ergodic Capacity of MIMO Channel in Reverberation Chamber
}

\author{
Xiaoming Chen \\ Department of Signals and Systems, Chalmers University of Technology, 41296 Gothenburg, Sweden \\ Correspondence should be addressed to Xiaoming Chen, xiaoming.chen@chalmers.se
}

Received 27 July 2011; Accepted 26 September 2011

Academic Editor: Li Yang

Copyright () 2012 Xiaoming Chen. This is an open access article distributed under the Creative Commons Attribution License, which permits unrestricted use, distribution, and reproduction in any medium, provided the original work is properly cited.

It has previously been shown that a reverberation chamber can conveniently be used to measure ergodic multiple-input multipleoutput (MIMO) capacity for over-the-air (OTA) tests. However, the MIMO channel in the reverberation chamber has not been fully studied before. In this paper, the spatial correlation of the MIMO channel in the chamber is studied by comparing the measured channel with two popular MIMO channel models. It is shown that the models can accurately predict the ergodic MIMO capacity of the channel in the reverberation chamber, but not the outage capacity (especially at high signal-to-noise regime). It is verified that the capacity estimation error is due to the fact that the measured MIMO channel in the chamber does not satisfy multivariate normality (MVN), which causes the capacity error increases additively with MIMO size and multiplicatively with signal-to-noise (SNR).

\section{Introduction}

Multiple-input multiple-output (MIMO) systems have drawn considerable popularity, over the past decade, due to their performance-enhancement capability in multipath environments [1]. Lots of work has been carried out for measuring MIMO capacity in real-life (outdoor and indoor) multipath environments [2-6]. As opposite to the real-life measurements, the reverberation chamber is being considered as a strong candidate for standardization of over-the-air (OTA) measurements for characterization of MIMO terminals due to its fast, repeatable, and economical measurements. The ergodic MIMO capacity can be measured in reverberation chambers [7-11]. While most of the reverberation chamber works have been focused on characterizing multiport antennas, the MIMO channel in the chamber has not been fully studied yet.

The aim of this paper is to study the measured channel in the chamber by comparing it with two well-known channel models, (i.e., Kronecker model and full-correlation model [1]) and physically explain the models' discrepancies with measurements. Some different properties of reverberation chamber and real-life multipath environments are also discussed. Most previous real-life measurements only used Kronecker model to compare with measurements [2-6]. Although there exist other sophisticated models, it is well known that the full-correlation model offers the best accuracy at the expanse of the most complexity. Therefore, we include both Kronecker model and the full-correlation model in this paper. It is found that both models have the same performance in terms of capacity estimation, which is a bit surprising. The reasons for this are discussed in Section 4. It is also found that, although both models can well predict the ergodic capacity (with only slight overestimation at high SNR regime), they fail to estimate the outage capacity, or the cumulative distribution function (CDF) of the capacity. Moreover, instead of capacity underestimations as indicated by real-life measurements [4], the present paper shows that Kronecker model tends to overestimate capacities based on measurements in a reverberation chamber. The reasons for this are discussed in Section 5.

This work is of particular interest for OTA characterization of MIMO terminals in reverberation chambers, because it helps to understand the channel conditions under which the passive and/or active MIMO measurements have been conducted in the chamber. 


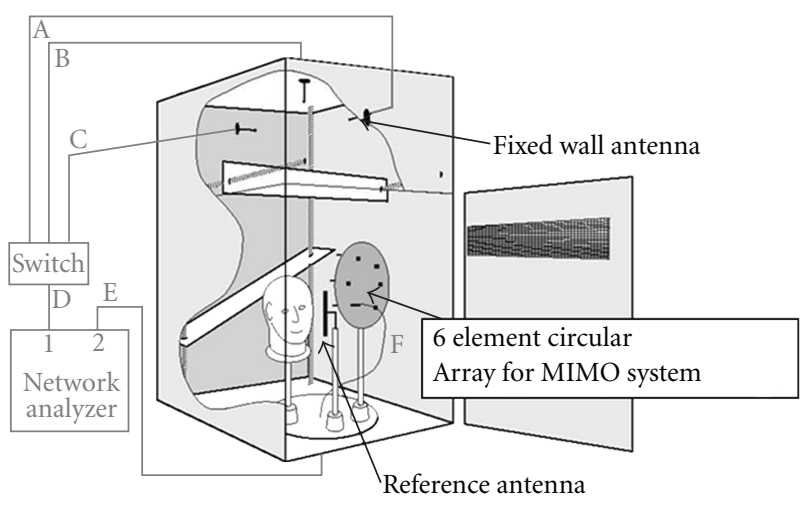

FIgure 1: Drawing of the Bluetest RC with two mechanical plate stirrers, platform, three wall antennas, and six-monopole array.

\section{Measurement Description}

It has been shown that the ergodic MIMO capacity of a multiantenna system can be easily determined based on the reverberation chamber measurement [7, 9-11]. The reverberation chamber is basically a metal cavity with many excited modes that are stirred to create a Rayleighfading environment [12]. The chamber used in the present paper is Bluetest HP reverberation chamber with a size of $1.75 \times 1.25 \times 1.8 \mathrm{~m}^{3}$ (see Figure 1 ). It has two plate mode stirrers, a turn-table platform, and three antennas mounted on three different walls (referred to as wall antennas thereafter). The wall antennas are actually wideband halfbow-tie (or triangular sheet) antennas. In the measurements, the platform (with a radius of $0.3 \mathrm{~m}$ ), on which the MIMO terminal under test was mounted, was moved to 20 positions equally spaced by $18^{\circ}$, and, for each platform position, the two plates simultaneously moved to 10 positions (equally spanned on the total distances that they can move along the walls). All the mechanical (stepwise) moves were controlled by a computer. At each stirrer position and for each wall antenna, a full frequency sweep was performed by the vector network analyzer (VNA), during which the channel transfer functions at different frequencies were sampled. The frequency step was set to $1 \mathrm{MHz}$ always. Therefore, for each transmit and receive antenna element pair, there are 200 channel transfer function samples per frequency point.

In order to calibrate out the long-term fading, or attenuation, in the chamber (so that only short-term fading came into play) [1], a reference measurement needed to be performed first, where the average power transfer function was measured using a reference antenna with known radiation efficiency. The reference level, $P_{\text {ref }}$, was obtained by dividing the average power function by the radiation efficiency of the reference antenna. Then, the multiport antenna under test, in this case a six-monopole array (see also Figure 1), was measured (actually this procedure was repeated for each of the monopole while the others are terminated with $50 \mathrm{ohm}$ ). During this measurement, the three wall antennas were assumed to be the transmit antenna elements; the monopole array was assumed to be the receive antenna. The monopoles have physical length of $8.3 \mathrm{~cm}$ (resonating at around $900 \mathrm{MHz}$ ). The ground plane has a radius of $14 \mathrm{~cm}$. The monopoles are uniformly mounted on the ground plane in a circle, where adjacent monopoles have a separation of $4.6 \mathrm{~cm}$. The small separation is necessary to have reasonably large correlations, in order to effectively compare different channel models [4].

The resulting channel matrix $\mathbf{H}_{6 \times 3}$ is a function of frequency and stirrer positions. For convenience, we introduce the following notation for the normalized measured channel matrix

$$
\mathbf{H}_{\text {meas }}=\frac{\mathbf{H}_{6 \times 3}}{\sqrt{P_{\text {ref }}}},
$$

where the reference level, $P_{\text {ref }}$, is described above. Note that the total radiation efficiency of the wall antenna is also calibrated out by (1).

Assume that the receiver has perfect channel state information and that transmitted power is equally allocated among transmitting antenna elements, the ergodic MIMO capacity can be computed from the measured channel matrices by [1]

$$
C=E\left\{\log _{2}\left[\operatorname{det}\left(\mathbf{I}+\frac{\gamma}{M_{T}} \mathbf{H}_{\text {meas }} \mathbf{H}_{\text {meas }}^{H}\right)\right]\right\},
$$

where $\gamma$ is signal-to-noise ratio (SNR) and the expectation $E$ can be approximated by averaging over all channel samples.

\section{MIMO Channel}

3.1. Channel Characterization. Wireless channel that can be assumed as wide-sense stationary uncorrelated scattering (WSSUS) [13] is usually characterized by its coherence bandwidth, coherence time, and coherence distance, or equivalently by their reciprocal counterparts: delay spread, Doppler spread, and angular spread, respectively [1]. Since the channel was sampled (by the VNA) stepwisely, that is, in static condition at each stirrer position, the Doppler spread of the channel is negligible. The angular distribution in the chamber is shown to be three-dimensional (3D) uniform, that is, isotropic [14]. The coherence bandwidth of the channel at the frequency of interest is around $1-2 \mathrm{MHz}$ [15]. In practice, wireless channels are seldom WSSUS. Fortunately, most of them can be assumed as quasi-WSSUS, that is, the channel statistics do not change within certain time and frequency intervals. These intervals are defined in [16] as stationarity time and stationarity bandwidth. Since channel was sampled under static condition, its stationarity time is infinite. Under the assumption of correlation underspread, the stationarity bandwidth is larger than $20 \mathrm{MHz}$ (i.e., at least 10 times larger than the coherence bandwidth). In the reverberation chamber, every subchannel (for each transmit and receive antenna element pair) is assumed to have the same time-frequency statistics.

Note that different fading-type environments can be emulated involving reverberation chamber. Holloway [17] showed that loading the chamber with electromagneticabsorbing objects can generate Rician fading. It was shown in [10] that two cascaded reverberation chambers can be 
used to emulate double-Rayleigh (key-hole) fading. More outdoor-like fading can be generated by connecting a reverberation chamber to an anechoic chamber [18]. As pointed out in [19], for MIMO channels, the most important aspect is the spatial correlation of the channel. Therefore, the main focus of this paper will be on the spatial correlation of the channel and its effects on capacity in an unloaded (with little electromagnetic-absorbing objects) reverberation chamber.

3.2. Channel Models. Assume a single-user narrow band MIMO system, consisting of $M_{T}$ transmit antennas and $M_{R}$ receive antennas, in a frequency flat Rayleigh-fading environment. The $M_{R} \times 1$ output vector of the MIMO system can be expressed as

$$
\mathbf{y}=\mathbf{H x}+\mathbf{n}
$$

where $\mathbf{H}$ is the $M_{T} \times M_{R}$ channel matrix, $\mathbf{x}$ is $M_{T} \times 1$ vector of transmit signals, and $\mathbf{n}$ is $M_{\mathrm{R}} \times 1$ zero mean white Gaussian noise vector. This paper will focus on narrowband models only, knowing that they can be readily extended to wideband model by assuming channel taps with different delays (with each tap modeled as a narrowband channel) [13].

A general, so-called, full-correlation channel model is given by [1]

$$
\operatorname{vec}\left(\mathbf{H}_{\text {full }}\right)=\mathbf{R}^{1 / 2} \operatorname{vec}\left(\mathbf{H}_{w}\right),
$$

where $\mathbf{H}_{w}$ is independent and identically distributed (i.i.d.) zero mean spatially white $M_{R} \times M_{T}$ channel matrix, $\operatorname{vec}(\mathbf{H})$ is the operator stacking the matrix $\mathbf{H}$ into a vector columnwise, $\mathbf{R}^{1 / 2}\left(\mathbf{R}^{1 / 2}\right)^{H}=\mathbf{R}$, and $\mathbf{R}$ is the covariance matrix that can be expressed as

$$
\mathbf{R}=E\left[\operatorname{vec}(\mathbf{H}) \operatorname{vec}(\mathbf{H})^{H}\right]
$$

where superscript ${ }^{H}$ is Hermitian operator. $\mathbf{H}_{w}$ is normalized so that its Frobenius norm satisfies $E\left[\left\|\mathbf{H}_{w}\right\|_{F}^{2}\right]=M_{T} M_{R}$. This normalization has the same physical meaning as (1), that is, path loss calibration. The full-correlation model is acknowledged as the most accurate model. However, it suffers from huge covariance matrix size and analytical intractability.

The Kronecker model assumes separable $\mathbf{R}$, that is,

$$
\mathbf{R}=\mathbf{R}_{t} \otimes \mathbf{R}_{r},
$$

where $\otimes$ denotes Kronecker product and $\mathbf{R}_{t}$ and $\mathbf{R}_{r}$ are covariance matrices at the transmit and receive sides, respectively. The superscript ${ }^{T}$ is transpose operator. Under this assumption, $\mathbf{H}$ can be represented using the so-called Kronecker model [1],

$$
\mathbf{H}_{\text {Kron }}=\mathbf{R}_{r}^{1 / 2} \mathbf{H}_{w} \mathbf{R}_{t}^{1 / 2} .
$$

This model has been verified in $[2,3,5]$ by real-life measurements. This model is relatively simple and analytically tractable. Furthermore, it allows independent optimizations of transmit and receive MIMO terminals. Therefore, the Kronecker model becomes the most popular MIMO channel model. However, it was shown in $[4,6]$ that Kronecker model leads to inaccurate capacity estimation. It was pointed out in [4] that the Kronecker model only rendered correct capacity when the antenna number at either side is no larger than three and that it tends to underestimate capacity otherwise.

\section{Measurement Results}

From Section 1, it is known that there are only 200 MIMO channel samples at one single frequency, which is too few to support reliable estimation. One simple way of increasing channel samples is to treat the channel samples at different frequencies as different channel realizations (or samples). This methodology has been used in [2] for reallife measurements. In a reverberation chamber, it is usually referred as frequency stirring or electronic stirring [20] (to increase independent samples). However, the frequency stirring bandwidth has to be carefully chosen so that more independent (or less correlated) samples can be included without changing the channel statistics. From Section 3, the coherence bandwidth of the channel is around $1-2 \mathrm{MHz}$, while the stationarity bandwidth is larger than $20 \mathrm{MHz}$. In practice, the antenna bandwidth will also affect the channel characteristics, since the (effective) channel also includes the antennas. Hence, the frequency stirring bandwidth should be larger than coherence bandwidth but smaller than stationarity bandwidth and antenna bandwidth. As a result, an empirical frequency stirring bandwidth of $8 \mathrm{MHz}$ (with $1-\mathrm{MHz}$ frequency step) is chosen, which is limited by the monopole bandwidth. As a result, there are 1600 MIMO channel samples for capacity evaluation.

The full channel covariance matrix is estimated from $\mathbf{H}_{\text {meas }}$ as

$$
\widehat{\mathbf{R}}=\frac{1}{N} \sum_{n=1}^{N} \operatorname{vec}\left(\mathbf{H}_{\text {meas }}\right) \operatorname{vec}\left(\mathbf{H}_{\text {meas }}\right)^{H}
$$

where $N=1600$ is the number of samples. Similarly, estimations of the covariance matrices at the transmit and receive sides are, respectively,

$$
\begin{gathered}
\hat{\mathbf{R}}_{t}=\frac{1}{N M_{R}} \sum_{n=1}^{N}\left(\mathbf{H}_{\text {meas }}^{H} \mathbf{H}_{\text {meas }}\right)^{T}, \\
\hat{\mathbf{R}}_{r}=\frac{1}{N M_{T}} \sum_{n=1}^{N} \mathbf{H}_{\text {meas }} \mathbf{H}_{\text {meas }}^{H} .
\end{gathered}
$$

Note that covariance matrices calculated using (8) and (9) include both antenna efficiencies and correlation coefficients. The corresponding ergodic capacity of the full-correlation model is

$$
\begin{gathered}
\widehat{C}_{\text {full }}=\frac{1}{N} \sum_{n=1}^{N}\left\{\log _{2}\left[\operatorname{det}\left(\mathbf{I}+\frac{\gamma}{M_{T}} \hat{\mathbf{H}}_{\text {full }} \hat{\mathbf{H}}_{\text {full }}^{H}\right)\right]\right\}, \\
\operatorname{vec}\left(\hat{\mathbf{H}}_{\text {full }}\right)=\hat{\mathbf{R}}^{1 / 2} \operatorname{vec}\left(\mathbf{H}_{w}\right) .
\end{gathered}
$$




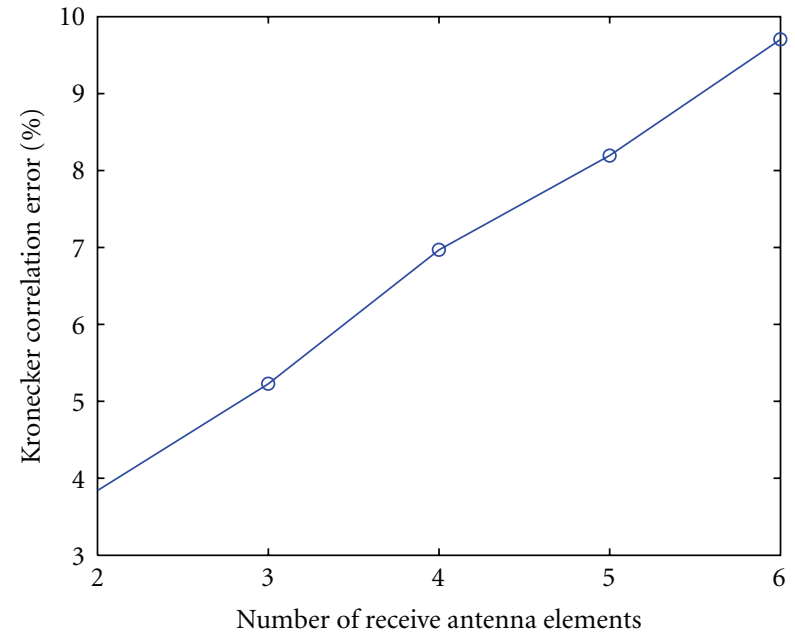

FIGURE 2: Kronecker correlation error as a function of receive monopole number with the three wall antennas fixed as transmit antenna.

Similarly, the corresponding ergodic capacity of the Kronecker model is

$$
\begin{gathered}
\hat{C}_{\text {Kron }}=\frac{1}{N} \sum_{n=1}^{N}\left\{\log _{2}\left[\operatorname{det}\left(\mathbf{I}+\frac{\gamma}{M_{T}} \hat{\mathbf{H}}_{\text {Kron }} \hat{\mathbf{H}}_{\text {Kron }}^{H}\right)\right]\right\}, \\
\hat{\mathbf{H}}_{\text {Kron }}=\hat{\mathbf{R}}_{r}^{1 / 2} \mathbf{H}_{w} \hat{\mathbf{R}}_{t}^{1 / 2} .
\end{gathered}
$$

Apart from comparing capacity, the discrepancy between the full-correlation and Kronecker models can be examined using the Kronecker correlation error defined in $[2,6]$,

$$
\Psi=\frac{\left\|\hat{\mathbf{R}}-\hat{\mathbf{R}}_{t} \otimes \hat{\mathbf{R}}_{r}\right\|_{F}}{\|\hat{\mathbf{R}}\|_{F}} .
$$

To examine the Kroneker model error as a function of the antenna element number, we fix the three wall antennas and increase the number of monopoles from two to six (the monopoles are always chosen from adjacent ones). The Kronecker correlation errors are then calculated and plotted in Figure 2. It can be seen that the Kronecker correlation model error increases with increasing receive monopole number. Similar result was shown in [2] based on real-life measurements.

From Figure 2, it is tempting to anticipate that the Kronecker model has worse capacity-estimation performance than the full-correlation model for a large antenna element number in a reverberation chamber, just as the cases for reallife multipath environments [4]. However, by comparing capacities based on the Kronecker model, full-correlation model, and measurements in the reverberation chamber (see Figures 3 and 4), it is shown that the Kronecker correlation error obtained in the chamber is a bit misleading in the sense that even when it is as high as $10 \%$, the capacities obtained using both channel models are almost the same, in spite of the deviation from measured ones at high SNR regime.

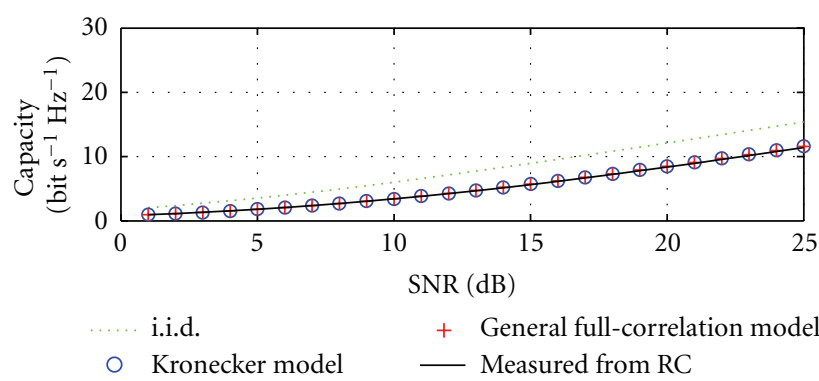

(a)

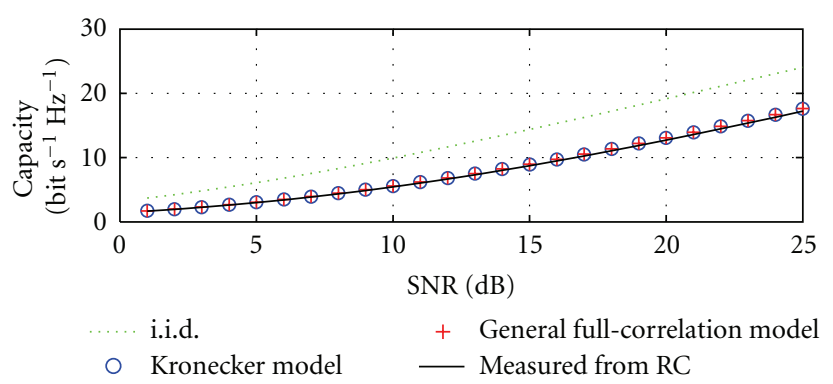

(b)

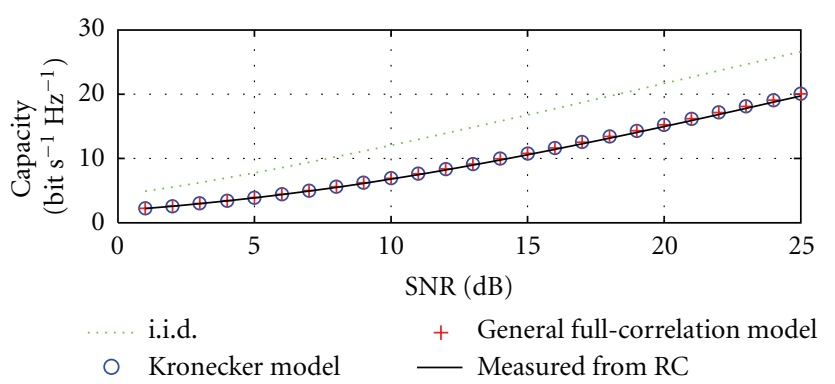

(c)

FIGURE 3: Comparison of the Kronecker model and full-correlation model against reverberation chamber measurement of $2 \times 3$ (a), $4 \times 3$ (b), and $6 \times 3$ (c) MIMO ergodic capacities, all at $900 \mathrm{MHz}$.

Figure 3 shows the comparison of ergodic capacities of the Kronecker and full-correlation models against measurements for the $2 \times 3$ (two adjacent monopoles and three wall antennas), $4 \times 3$ (four adjacent monopoles and three wall antennas), and $6 \times 3$ (all six monopoles and three wall antennas) MIMO systems. As can be seen, the Kronecker model gives almost identical ergodic capacity as the fullcorrelation model; both models well predict ergodic capacity from measured channel in the chamber. Both Kronecker and full-correlation models slightly overestimate the measured ergodic capacity as the monopole number exceeds three. The ergodic capacity of the corresponding i.i.d. channels are also plotted for each case. The capacity degradation of the measurement from the corresponding i.i.d. channel is due to antenna correlations and efficiencies.

Figure 4 shows the CDFs of the capacities from the Kronecker model, full-correlation model, and measurements for the same $2 \times 3,4 \times 3$, and $6 \times 3$ MIMO systems with SNRs 


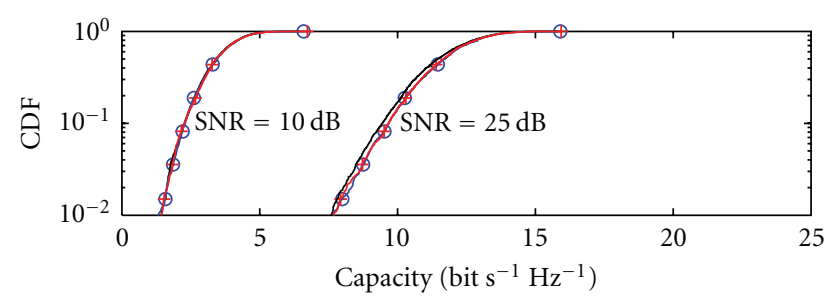

○ Kronecker model _ _ Measurement

+ Full-correlation model

(a)

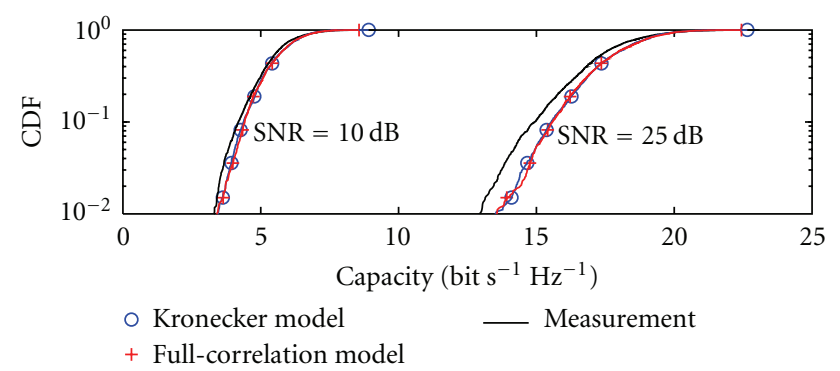

(b)

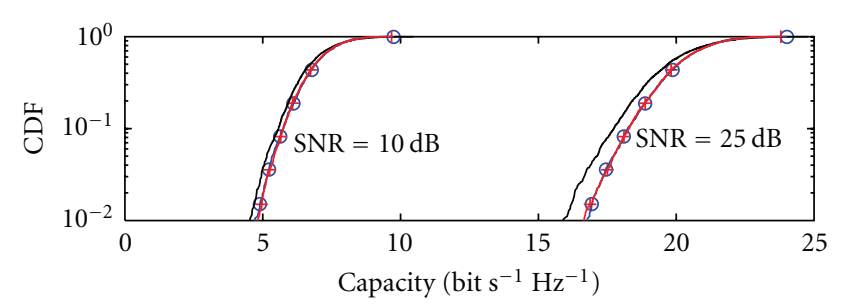

○ Kronecker model

+ Full-correlation model

(c)

FIGURE 4: Comparison of the Kronecker model and full-correlation model against reverberation chamber measurement of $2 \times 3$ (a), $4 \times 3$ (b), and $6 \times 3$ (c) CDF of MIMO capacities, with SNR $=10 \mathrm{~dB}$ and $25 \mathrm{~dB}$, all at $900 \mathrm{MHz}$.

of $10 \mathrm{~dB}$ and $25 \mathrm{~dB}$. For all cases, it is seen that the Kronecker and full-correlation models give almost the same result. However, both models overestimate the measured capacity CDFs for more than three antenna elements, especially at high SNR regime, opposite to the capacity underestimation of the Kronecker model observed in [4] based on real-life measurements. The capacity overestimation of the Kronecker model was also observed in [2] for a $3 \times 3$ MIMO system based on real-life measurements. Note that it is shown in [21] that Kronecker model could also overestimate capacity.

It is shown that the Kronecker model has the same performance as the full-correlation model because that multibounce rich scattering property of the chamber makes the correlations at transmit and receive sides separable [22]. And, due to this property, the reverberation chamber can be used to characterize the MIMO performance of a MIMO terminal independent of the other MIMO end, which is highly desired in OTA MIMO terminal tests.

\section{Multivariate Normality Test}

The good agreement between the Kronecker model and the other two advanced models means that, unlike in real-life multipath environments, the discrepancy of capacity of the Kronecker model with the measured one in the reverberation chamber is not due to the Kronecker structure. Instead, it is probably because the entries of the measured MIMO channel matrix are not jointly Gaussian (or normal). It has been found that the Henze-Zirkeler's test [23] has a good overall power against other alternatives to $\operatorname{MVN}[6,24]$. Therefore, it is applied to the measured channel matrices in the chamber.

Let $\mathbf{h}_{n}=\operatorname{vec}\left(\mathbf{H}_{\text {meas }}\right)$ be the vector of the $n$th channel sample, then the test statistic is

$$
\begin{aligned}
T= & \frac{1}{N} \sum_{n, m=1}^{N} \exp \left[-\frac{\beta^{2}}{2}\left(\mathbf{h}_{n}-\mathbf{h}_{m}\right)^{H} \widehat{\mathbf{R}}^{-1}\left(\mathbf{h}_{n}-\mathbf{h}_{m}\right)\right] \\
& -2\left(1+\beta^{2}\right)^{-M_{T} M_{R} / 2} \\
& \times \sum_{n=1}^{N} \exp \left[-\frac{\beta^{2}}{2\left(1+\beta^{2}\right)}\left(\mathbf{h}_{n}-\overline{\mathbf{h}}\right)^{H} \widehat{\mathbf{R}}^{-1}\left(\mathbf{h}_{n}-\overline{\mathbf{h}}\right)\right] \\
& +N\left(1+\beta^{2}\right)^{-M_{T} M_{R} / 2}
\end{aligned}
$$

where

$$
\begin{aligned}
& \beta=\frac{1}{\sqrt{2}}\left[\frac{N\left(2 M_{T} M_{R}+1\right)}{4}\right]^{1 / M_{T} M_{R}+4}, \\
& \overline{\mathbf{h}}=\frac{1}{N} \sum_{n}^{N} \mathbf{h}_{n} .
\end{aligned}
$$

The mean and variance of $T$ are, respectively,

$$
\begin{aligned}
\mu= & 1-\left(1+2 \beta^{2}\right)^{-M_{T} M_{R} / 2} \\
& \times\left[1+\frac{M_{T} M_{R} \beta^{2}}{1+2 \beta^{2}}+\frac{M_{T} M_{R}\left(M_{T} M_{R}+2\right) \beta^{4}}{\left(1+2 \beta^{2}\right)^{2}}\right], \\
\sigma^{2}= & 2\left(1+4 \beta^{2}\right)^{-M_{T} M_{R} / 2}+2\left(1+2 \beta^{2}\right)^{-M_{T} M_{R}} \\
& \times\left[1+\frac{2 M_{T} M_{R} \beta^{4}}{\left(1+2 \beta^{2}\right)^{2}}+\frac{3 M_{T} M_{R}\left(M_{T} M_{R}+2\right) \beta^{8}}{4\left(1+2 \beta^{2}\right)^{4}}\right] \\
& \left.-4 w^{-M_{T} M_{R} / 2}\right] \\
& \times\left[1+\frac{3 M_{T} M_{R} \beta^{4}}{2 w}+\frac{M_{T} M_{R}\left(M_{T} M_{R}+2\right) \beta^{8}}{2 w^{2}}\right] .
\end{aligned}
$$

Let the null hypothesis be that $\mathbf{H}_{\text {meas }}$ is MVN, that is, the test statistic $T$ is approximately lognormally distributed. The probability that the null hypothesis is rejected although it is true is called significance level [25]. If the complementary CDF (CCDF) at $T$ for a lognormal distribution ( $P$ value),

$$
P=\frac{1}{\sqrt{2 \pi \sigma^{2}}} \int_{T}^{\infty} \frac{1}{t} \exp \left[-\frac{(\ln t-\mu)^{2}}{2 \sigma^{2}}\right] d t,
$$


TABle 1: $P$ value of Henze-Zirkeler's test.

\begin{tabular}{lccc}
\hline & Number of wall antennas: 1 & Number of wall antennas: 2 & Number of wall antennas: 3 \\
\hline Number of Monopoles: 1 & 0.850 & 0.196 & 0.000 \\
Number of Monopoles: 2 & 0.657 & 0.060 & 0.000 \\
Number of Monopoles: 3 & 0.370 & 0.000 & 0.000 \\
Number of Monopoles: 4 & 0.230 & 0.000 & 0.000 \\
Number of Monopoles: 5 & 0.066 & 0.000 & 0.000 \\
Number of Monopoles: 6 & 0.020 & 0.000 & 0.000 \\
\hline
\end{tabular}

is no smaller than the significance level, $\mathbf{H}_{\text {meas }}$ satisfies MVN. Otherwise, $\mathbf{H}_{\text {meas }}$ does not satisfy MVN. A good significance level for Henze-Zirkeler's MVN test is found to be 0.05 [25], which is also used in this paper.

Applying the Henze-Zirkeler's test to the measured channel matrices, it is found that every subchannel coefficient of $\mathbf{H}_{\text {meas }}$ is still in (complex) normal distribution, but they are together not jointly normal, that is, $\mathbf{H}_{\text {meas }}$ does not satisfy MVN. This finding is a bit surprising, since it is well known the single-input single-output (SISO) channel in a reverberation chamber is in (complex) normal distribution [12]. And it is usually assumed that MIMO channels in the chamber are jointly normal. To find out what is the reason of the nonMVN, the submatrices of $\mathbf{H}_{\text {meas }}$ are tested separately. The corresponding $P$ values are listed in Table 1 . By comparing the $P$ values with the significant level (0.05), it is found that the subchannel vectors of the monopoles (for one wall antenna) are jointly normal up to five monopole elements, while the subchannel vectors of the wall antennas (for one monopole) are only jointly normal for two wall antennas, that is, the subchannels of the three wall antennas not jointly normal.

It is found that the channels with large MIMO sizes show strong non-MVN and that channels with MIMO small size (e.g., $2 \times 2$ ) approximately satisfy MVN. The different MVN properties for the monopole array and wall antennas are due to the fact that the monopole array is uniformly and circularly distributed (with the same polarization), while the wall antennas are located arbitrarily on three different walls with orthogonal polarizations (which is necessary to keep a desired polarization balance). Thus, the monopole array satisfies antenna stationarity (i.e., antenna correlation depends only on the antenna element separation, not the element positions) [19], while wall antennas do not. The antenna stationarity of an antenna array enables its correlation matrix to converge, which guarantees MVN of the channels seen by the array (cf. weakly converging Gaussian vectors theorem [26]). Note that a well-stirred reverberation chamber is polarization balanced, that is, there is no polarization preference in the chamber. Thus, there is no need to introduce a polarization matrix to the Kronecker model as for the polarization-unbalanced cases [1].

Due to the non-MVN of $\mathbf{H}_{\text {meas }}$, neither of the abovementioned channel models can estimate the MIMO capacity accurately, because all of them involve $\mathbf{H}_{w}$ (i.i.d. zero mean complex Gaussian matrix). It can be seen from the MIMO capacity formula (2) that the channel model error is additively increased by MIMO size and multiplicatively increased by SNR. This explains why the capacity estimation error increases with either increasing MIMO size or increasing SNR. And, therefore, the non-MVN is the main contribution to capacity estimation errors of the channel models in the reverberation chamber. It seems that the only way to circumvent this problem is to give up the MVN assumption in channel modeling. However, the ultimate goal of channel modeling is to find a model that can predict the actual channel correctly and, at the same time, can be used with reasonably low complexity from communication- and information-theoretic viewpoints. Since MVN is virtually the only mathematically tractable multivariate distribution [26], any model giving up the MVN assumption might not be very much useful from communication- and informationtheoretic point of views, even though it might offer better estimation accuracy. Therefore, no effort is exerted in finding a model with better accuracy in this paper.

Note that the non-MVN may not be the main contribution for channel model errors for real-life multipath environments, where the full-correlation model can outperform the Kronecker model. The equal performance of all the models for the reverberation chamber measurement is because of the multibounce rich scattering property of the chamber, as discussed in Section 4.

\section{Conclusion}

In this paper, the MIMO channel in a reverberation chamber is studied by comparing the measurements with different channel models. It is found that both models have the same performance in terms of capacity estimation for the reverberation chamber measurements and that all of them can well predict the ergodic capacity up to six antenna elements with only slight overestimation at high-SNR regime. However, the models fail to predict the CDFs of the capacities for more than three antenna elements, especially at high SNR regime. The reason for this is because of the non-MVN of the MIMO channel in the chamber. Since all the models involves i.i.d. complex Gaussian channel, there will be modeling errors due to the non-MVN of the measured channel. And the channel modeling errors will additively increase with MIMO size and multiplicatively increase with SNR, for MIMO capacity estimations. The equal performance of the Kronecker model and the full-correlation model (for reverberation chamber measurements) implies that the correlations at transmit and receive sides can be treated separately. This is actually very desirable, since it allows characterizing the performance of 
a MIMO terminal independently (without the effect of the other MIMO side) by doing an OTA test in the chamber, which in turn allows fair comparisons of different MIMO terminals.

\section{Acknowledgments}

This work has been supported by The Swedish Governmental Agency for Innovation Systems (VINNOVA) within the VINN Excellence Center Chase. The author would like to thank Dr. Li Yang and the anonymous reviewers for their helpful comments to increase the quality of this paper.

\section{References}

[1] A. Paulraj, R. Nabar, and D. Gore, Introduction to Space-Time Wireless Communication, Cambridge University Press, Cambridge, Mass, USA, 2003.

[2] K. Yu, M. Bengtsson, B. Ottersten, D. McNamara, P. Karlsson, and M. Beach, "Modeling of wide-band MIMO radio channels based on NLoS indoor measurements," IEEE Transactions on Vehicular Technology, vol. 53, no. 3, pp. 655-665, 2004.

[3] J. P. Kermoal, L. Schumacher, K. I. Pedersen, P. E. Mogensen, and F. Frederiksen, "A stochastic MIMO radio channel model with experimental validation," IEEE Journal on Selected Areas in Communications, vol. 20, no. 6, pp. 1211-1226, 2002.

[4] H. Özcelik, M. Herdin, W. Weichselberger, J. Wallace, and E. Bonek, "Deficiencies of "Kronecker" MIMO radio channel model," Electronics Letters, vol. 39, no. 16, pp. 1209-1210, 2003.

[5] D. Chizhik, J. Ling, P. W. Wolniansky, R. A. Valenzuela, N. Costa, and K. Huber, "Multiple-input-multiple-output measurements and modeling in Manhattan," IEEE Journal on Selected Areas in Communications, vol. 21, no. 3, pp. 321-330, 2003.

[6] T. Svantesson and J. W. Wallace, "Tests for assessing multivariate normality and the covariance structure of MIMO data," in Proceedings of the IEEE International Conference on Accoustics, Speech, and Signal Processing, Hong Kong, April 2003.

[7] K. Rosengren and P.-S. Kildal, "Radiation efficiency, correlation, diversity gain and capacity of a six-monopole antenna array for a MIMO system: theory, simulation and measurement in reverberation chamber," in Proceedings of the IEEE Microwaves, Optics and Antenna, vol. 152, pp. 7-16, February 2005.

[8] J. F. Valdés, M. A. Fernandez, A. M. Gonzalez, and D. A. Hernandez, "The influence of efficiency on receive diversity and MIMO capacity for Rayleigh-fading channels," IEEE Transactions on Antennas and Propagation, vol. 56, no. 5, pp. 1444-1450, 2008.

[9] L. Garcia-Garcia, B. Lindmark, N. Jaldén, and C. Orlenius, "MIMO capacity of antenna arrays evaluated using radio channel measurements, reverberation chamber and radiation patterns," IET Microwaves, Antennas and Propagation, vol. 1, no. 6, pp. 1160-1169, 2007.

[10] O. Delangre, P. de Doncker, M. Lienard, and P. Degauque, "Coupled reverberation chambers for emulating MIMO channels," Comptes Rendus Physique, vol. 11, no. 1, pp. 30-36, 2010.

[11] X. Chen, P.-S. Kildal, J. Carlsson, and J. Yang, "Comparison of ergodic capacities from wideband MIMO antenna measurements in reverberation chamber and anechoic chamber," IEEE Antennas and Wireless Propagation Letters, vol. 10, pp. 446449, 2011.
[12] J. G. Kostas and B. Boverie, "Statistical model for a modestirred chamber," IEEE Transactions on Electromagnetic Compatibility, vol. 33, no. 4, pp. 366-370, 1991.

[13] R. Vaughan and J. B. Andersen, Channels, Propagation and Antennas for Mobile Communications, Intelligent Energy Europe, London, UK, 2003.

[14] D. A. Hill, "Plane wave integral representation for fields in reverberation chambers," IEEE Transactions on Electromagnetic Compatibility, vol. 40, no. 3, pp. 209-217, 1998.

[15] X. Chen, P.-S. Kildal, C. Orlenius, and J. Carlsson, "Channel sounding of loaded reverberation chamber for over-the-air testing of wireless devices: coherence bandwidth versus average mode bandwidth and delay spread," IEEE Antennas and Wireless Propagation Letters, vol. 8, pp. 678-681, 2009.

[16] G. Matz, "Characterization of non-WSSUS fading dispersive channels," in Proceedings of the International Conference on Communications (ICC '03), pp. 2480-2484, Anchorage, Alaska, USA, May 2003.

[17] C. L. Holloway, D. A. Hill, J. M. Ladbury, P. F. Wilson, G. Koepke, and J. Coder, "On the use of reverberation chamber to simulate a rician radio environment for the testing of wireless devices," IEEE Transactions on Antennas and Propagation, vol. 54, no. 11, pp. 3167-3177, 2006.

[18] H. Fielitz, K. A. Remley, C. L. Holloway, Q. Zhang, Q. Wu, and D. W. Matolak, "Reverberation-chamber test environment for outdoor urban wireless propagation studies," IEEE Antennas and Wireless Propagation Letters, vol. 9, Article ID 5406096, pp. 52-56, 2010.

[19] M. Herdin, Non-stationary indoor MIMO radio channels, Ph.D. thesis, Vienna University of Technology, Vienna, Austria, 2004.

[20] D. A. Hill, "Electronic mode stirring for reverberation chambers," IEEE Transactions on Electromagnetic Compatibility, vol. 36, no. 4, pp. 294-299, 1994.

[21] V. Raghavan, J. H. Kotecha, and A. M. Sayeed, "Why does the Kronecker model result in misleading capacity estimates?" IEEE Transactions on Information Theory, vol. 56, no. 10, Article ID 5571876, pp. 4843-4864, 2010.

[22] A. S. Y. Poon, D. N. C. Tse, and R. W. Brodersen, "Impact of scattering on the capacity, diversity, and propagation range of multiple-antenna channels," IEEE Transactions on Information Theory, vol. 52, no. 3, pp. 1087-1100, 2006.

[23] N. Henze and B. Zirkler, "A class of invariant consistent tests for multivariate normality," Communications in StatisticsTheory and Methods, vol. 19, no. 10, pp. 3595-3618, 1990.

[24] A. Trujillo-Ortiz, R. Hernandez-Walls, K. Barba-Rojo, and L. Cupul-Magana, HZmvntest: Henze-Zirkler's Multivariate Normality Test, 2007.

[25] R. B. Agostino and M. A. Stephen, Goodness-of-Fit Techniques, Marcel Dekker, New York, NY, USA, 1986.

[26] I. I. Gikhman and A. V. Skorokhod, Introduction to the Theory of Random Processes, Dover publication, New York, NY, USA, 1996. 

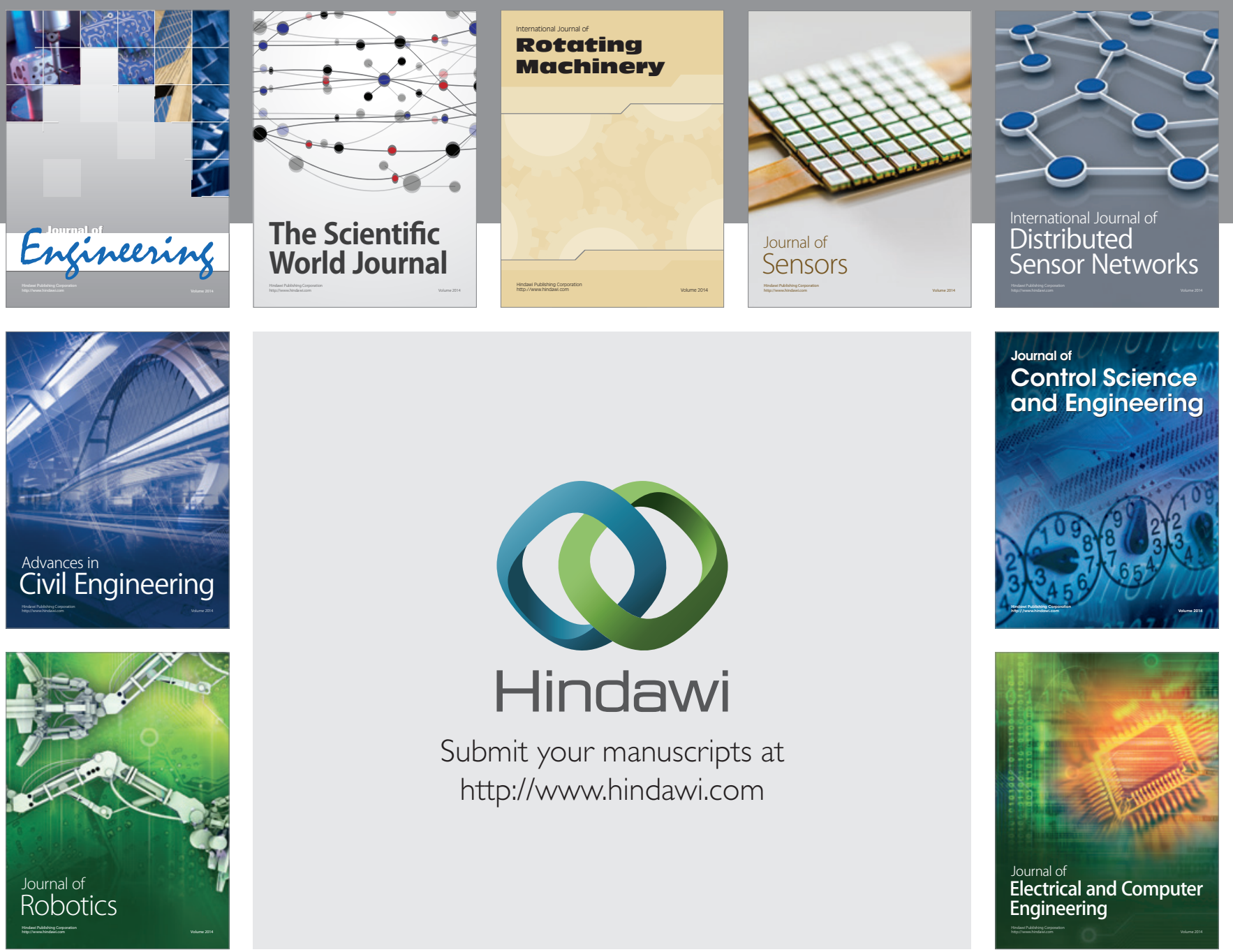

Submit your manuscripts at

http://www.hindawi.com
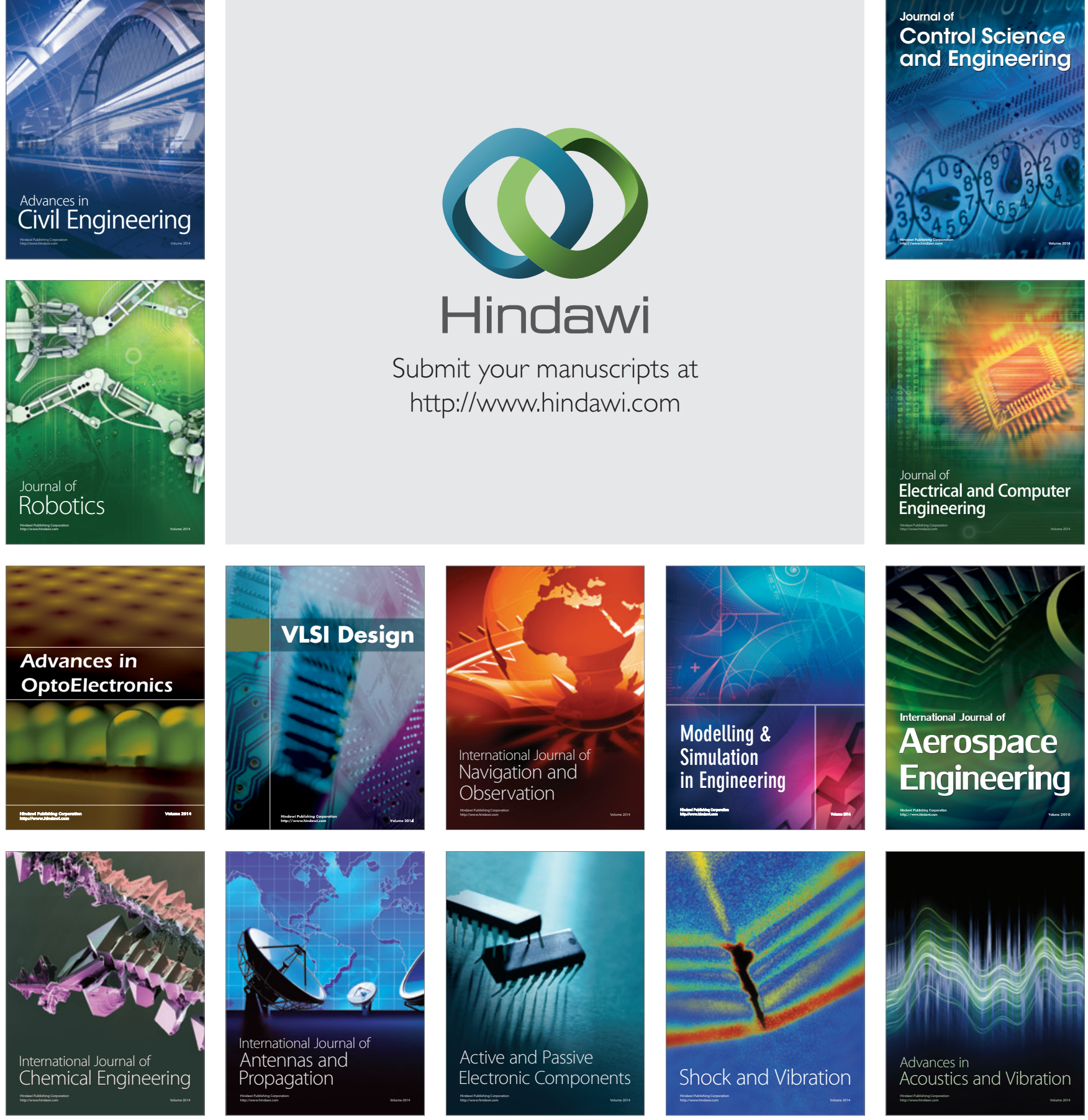